\title{
Conservative Control Systems Described by the Schrödinger Equation
}

\author{
Salah E. Rebiai \\ Department of Mathematics, Faculty of Sciences, \\ University of Batna, 05000 Batna, Algeria \\ rebiai@hotmail.com
}

\begin{abstract}
An important subclass of well-posed linear systems is formed by the conservative systems. A conservative system is a system for which a certain energy balance equation is satisfied both by its trajectories and those of its dual system. In Malinen et al. [10], a number of algebraic characterizations of conservative linear systems are given in terms of the operators appearing in the state space description of the system. Weiss and Tucsnak [20] identified by a detailed argument a large class of conservative linear systems described by a second order differential equation in a Hilbert space and an output equation, and they may have unbounded control and observation operators. In this paper, we give two examples of conservative linear control systems described by the linear Schrödinger equation on an $n$-dimensional domain with boundary control and boundary observation. These examples do not fit into the framework of [20].
\end{abstract}

\section{Introduction}

Abstract representation of particular classes of infinite dimensional systems has received considerable attention in the literature. For partial differential equations subject to control either acting on the boundary of, or else as a point control within, a multidimensional bounded domain we refer to Balakrishnan [1] and Washburn [17] for parabolic problems and to Triggiani [15], Lasiecka and Triggiani [4], Flandoli et al. [3] for hyperbolic and Petrowsky-type problems. For functional differential equations with delays in control and observation, see Salamon [12] and Delfour [2]. In [13], Salamon introduced the class of well-posed infinite dimensional linear systems. The aim was to provide a unifying abstract framework to formulate and solve control problems for systems described by functional and partial differential equations. Roughly speaking, a well-posed linear system is a linear time invariant system such that on any finite time interval, the operator from the initial state and the input function to the final state and the output function is bounded. An important subclass of well-posed linear systems is formed by the conservative systems. A conservative system is a system for which a certain energy balance equation is satisfied both by its trajectories and those of its dual system. In Malinen et al. [10], a number of algebraic characterizations of conservative linear systems are given in terms of the operators appearing in the state space description of the system.

Weiss and Tucsnak [20] identified by a detailed argument a large class of conservative linear systems described by a second order differential equation in a Hilbert space

A. Korytowski et al. (Eds.): System Modeling and Optimization, IFIP AICT 312, pp. 448-458, 2009.

(c) IFIP International Federation for Information Processing 2009 
and an output equation, and they may have unbounded control and observation operators.

In this paper, we give two examples of conservative control systems described by the linear Schrödinger equation with boundary control and boundary observation. These examples do not fit into the framework of [20].

The following notations are used. Let $X$ be a Hilbert space, then:

- $C^{n}(0,+\infty ; X)$ : the space of $n$ times continuously differentiable $X$-valued functions on $[0,+\infty), n \in \mathbb{N}$.

- $B C^{n}(0,+\infty ; X)$ : the space of those $f \in C^{n}(0,+\infty ; X)$ for which $f, f^{\prime}, \ldots, f^{(n)}$ are all bounded on $[0,+\infty)$.

- $H^{n}(0,+\infty ; X)$ : the Sobolev space of $X$-valued functions with $n \in \mathbb{N}$.

\section{Some Concepts from Conservative Well-Posed Linear Systems}

In this section, we first gather some basic facts about admissible control and observation operators and about well-posed linear systems. For more details we refer to Falandoli et al. [3] (though here the expressions "admissible" and "well-posed" are not stated explicitly), Salamon [13], Weiss [18] and Staffans [14]. Then, we recall the definition and some basic properties of conservative systems, see Weiss et al. [19], Tucsnak and Weiss [16], Weiss and Tucsnak [20] for further details.

Let $X$ be a Hilbert space and $A: D(A) \rightarrow X$ be the generator of a strongly continuous semigroup $\mathbb{S}$ on $X$. We define the Hilbert space $X_{1}$ as $D(A)$ with the norm $\|y\|_{1}=$ $\|(\beta I-A) y\|$ where $\beta \in \rho(A)$ is fixed. The Hilbert space $X_{-1}$ is the completion of $X$ with respect to the norm $\|y\|_{-1}=\left\|(\beta I-A)^{-1} y\right\|$. It is known that $X_{-1}=D\left(A^{*}\right)^{\prime}$, the dual space with respect to the pivot space $X$. We have

$$
X_{1} \subset X \subset X_{-1}
$$

with continuous dense injections.

$\mathbb{S}$ extends to a semigroup on $X_{-1}$ denoted by the same symbol. The generator of this semigroup is an extension of $A$ whose domain is $X$ so that $A: X \rightarrow X_{-1}$.

Definition 1. Let $U$ be a Hilbert space. The operator $B$ is said to be an admissible control operator of $\mathbb{S}$ if the input maps $\left\{\Phi_{t}\right\}_{t \geq 0}$ are bounded from $L^{2}(0,+\infty ; U)$ to $X$ for all finite $t \geq 0$ where

$$
\Phi_{t} u:=\int_{0}^{t} \mathbb{S}(t-\tau) B u(\tau) d \tau
$$

If $y$ is a solution of

$$
y^{\prime}(t)=A y(t)+B u(t), t \geq 0,
$$

which is an equation in $X_{-1}$ with $y(0) \in X$ and $u \in L^{2}(0,+\infty ; U)$, then $y(t) \in X$ for all $t \geq 0$. In this case $y$ is a continuous $X$-valued function of $t$ and we have for all $t \geq 0$

$$
y(t)=\mathbb{S}(t) y(0)+\int_{0}^{t} \mathbb{S}(t-\tau) B u(\tau) d \tau
$$


The admissible control operator is called infinite-time admissible if for any $u \in$ $L^{2}(0,+\infty ; U)$ the map

$$
t \rightarrow \Phi_{t} u
$$

from $[0,+\infty)$ to $X$ is bounded.

Definition 2. Let $Y$ be another Hilbert space. The operator $C \in \mathscr{L}\left(X_{1}, Y\right)$ is called an admissible observation operator for $\mathbb{S}$ if for every $t>0$ there exists a $k_{t} \geq 0$ such that

$$
\int_{0}^{t}\left\|C \mathbb{S}(\tau) y_{0}\right\|^{2} d \tau \leq k_{t}\left\|y_{0}\right\|^{2}, \forall y_{0} \in D(A)
$$

The admissibility of $C$ means that there is a continuous operator

$$
\Psi: X \rightarrow L_{l o c}^{2}(0,+\infty ; Y)
$$

such that

$$
\left(\Psi y_{0}\right)(t)=C \mathbb{S}(t) y_{0}, \forall y_{0} \in D(A) .
$$

The operator $\Psi$ is completely determined by $(8)$ because $D(A)$ is dense in $X$.

$C$ is said to be an infinite-time admissible observation operator for $\mathbb{S}$ if there exists $K>0$ such that

$$
\int_{0}^{+\infty}\left\|C \mathbb{S}(\tau) y_{0}\right\|^{2} d \tau \leq K\left\|y_{0}\right\|^{2}, \forall y_{0} \in D(A) .
$$

The following duality result holds.

Theorem 1. (Salamon [13], Staffans [14]) $C$ is an (infinite-time) admissible observation operator for $\mathbb{S}$ if and only if $C^{*}$ is an (infinite-time) admissible control operator for the adjoint semigroup $\mathbb{S}^{*}$.

Remark 1. In view of the above theorem, we see that Definition 1 is equivalent to the Hypothesis (H1) in Flandoli et al. [3].

Remark 2. For PDE systems with boundary control such as the multidimensional Schrödinger equation with Dirichlet control, the admissibility of $B$ is a sharp trace regularity result not obtainable by the standard trace theory. It is established by PDE hard analysis energy methods (see Lasiecka and Triggiani [5] and [6] and the references therein).

Theorem 2. If $B \in \mathscr{L}\left(U, X_{-1}\right)$ is an admissible control operator for $\mathbb{S}$ and $C \in$ $\mathscr{L}\left(X_{1}, Y\right)$ is an admissible operator for $\mathbb{S}$, then the transfer functions of the system $\Sigma$ given by the triple $(A, B, C)$ are solutions of

$$
G: \rho(A) \rightarrow \mathscr{L}(U, Y)
$$

of

$$
G(s)-G(\beta)=-(s-\beta) C(s I-A)^{-1}(\beta I-A)^{-1} B
$$

for $s$ and $\beta$ in $\rho(A)$. 
Definition 3. The system $\Sigma$ given by the triple $(A, B, C)$ is said to be a well-posed linear system if $B \in \mathscr{L}\left(U, X_{-1}\right)$ is an admissible control operator for $\mathbb{S}$ and $C \in \mathscr{L}\left(X_{1}, Y\right)$ is an admissible operator for $\mathbb{S}$, and its transfer functions are bounded on some right-half plane.

For a well-posed linear system the operator $\Sigma_{t}$ from the initial state and the input function to the final state and the output function is bounded on any finite time interval $[0, t]$. The input and output functions $u$ and $z$ are locally $L^{2}$ with values in $U$ and $Y$ respectively. The state trajectory $y$ is an $X$-valued function.

The boundedness property mentioned above means that for every $t>0$, there is a $c_{t}>0$ such that

$$
\|y(t)\|^{2}+\int_{0}^{t}\|z(\tau)\|^{2} d \tau \leq c_{t}\left(\|y(0)\|^{2}+\int_{0}^{t}\|u(\tau)\|^{2} d \tau\right) .
$$

Remark 3. The class of well-posed linear systems includes many systems described by partial differential equations or delay differential equations. The formal resemblance to finite dimensional systems is one of its main advantages. Much work has been done on this class of systems, see Staffans [14] and the references therein. There are however important systems that do not belong to this class, see Lasiecka and Triggiani [67].

Definition 4. A well-posed linear system $\Sigma$ given by the triple $(A, B, C)$ is called conservative if for every $t \geq 0$, the operator

$$
\Sigma_{t}: X \times L^{2}(0, t ; U) \rightarrow X \times L^{2}(0,+\infty ; Y)
$$

is unitary. This means that for every $t \geq 0$, the following statements are true:

(i) $\Sigma_{t}$ is an isometry, i.e.

$$
\|y(t)\|^{2}+\int_{0}^{t}\|z(\tau)\|^{2} d \tau=\|y(0)\|^{2}+\int_{0}^{t}\|u(\tau)\|^{2} d \tau
$$

(ii) $\Sigma_{t}$ is onto.

Theorem 3. (Weiss and Tucsnak [20]) The system $\Sigma$ is conservative if and only if the balance equation (14) or its differential form

$$
\frac{d}{d t}\|y(t)\|^{2}=\|u(t)\|^{2}-\|z(t)\|^{2}
$$

holds for all state trajectories of $\Sigma$ as well as for all state trajectories of the dual system $\Sigma_{d}$ for suitable initial state and input function.

In Tucsnak and Weiss [16], the authors investigated conditions under which such systems are exponentially stable or strongly stable. It turns out that these properties are equivalent to certain controllability and observability properties. 


\section{The Schrödinger Equation with Dirichlet-Type Boundary Feedback}

Let $\Omega$ be an open bounded domain in $\mathbb{R}^{n}$ with $C^{2}$-boundary $\Gamma=\bar{\Gamma}_{0} \cup \Gamma_{1}$ where $\Gamma_{0}$ and $\Gamma_{1}$ are disjoint parts of $\Gamma$ with $\Gamma_{1} \neq \varnothing$.

Let $G: H^{-1}(\Omega) \rightarrow H_{0}^{1}(\Omega)$ be the operator defined by:

$$
G f=\varphi \text { if and only if } \varphi \in H_{0}^{1}(\Omega) \text { and }-\Delta \varphi=f .
$$

We consider the system described by the equations

$$
\begin{gathered}
y_{t}(x, t)=\mathbf{i} \Delta y(x, t) \text { in } \Omega \times(0,+\infty) \\
y(x, 0)=y_{0}(x) \text { in } \Omega \\
y=0 \text { on } \Gamma_{0} \times(0,+\infty) \\
y+\frac{\mathbf{i}}{2} \frac{\partial(G y)}{\partial v}=u \text { on } \Gamma_{1} \times(0,+\infty) .
\end{gathered}
$$

In (20), $v=\left(v_{1}, \ldots, v_{n}\right)$ is the unit outward normal on $\Gamma$.

The input of this system is the function $u$ in (20). The output associated with this system is

$$
z=y-\frac{\mathbf{i}}{2} \frac{\partial(G y)}{\partial v} \text { on } \Gamma_{1} \times(0,+\infty) .
$$

Remark 4. The system (17)- without the term $\mathbf{i} \frac{\partial(G y)}{\partial v}$ has been considered in Lasiecka and Triggiani [5] where $H^{-1}(\Omega)$ is identified as the space of optimal regularity and exact controllability and uniform stabilization results have been established on this space, the latter via the dissipative feedback $u=-\mathbf{i} \frac{\partial(G y)}{\partial v}$.

The precise statement of well-posedness and conservativity of the system described by (17)-(21) is given in the following theorem.

Theorem 4. The equations (17)-(21) determine a conservative linear system $\Sigma$ with input and output space $U=L^{2}\left(\Gamma_{1}\right)$ and state space $X=H^{-1}(\Omega)$.

If

$$
y_{0} \in Z_{D}=\left\{f \in L^{2}(\Omega): \Delta f \in H^{-1}(\Omega),\left.f\right|_{\Gamma} \in L^{2}(\Gamma) \text { and } f=0 \text { on } \Gamma_{0}\right\}
$$

and the compatibility condition

$$
y_{0}(x)+\frac{\mathbf{i}}{2} \frac{\partial\left(G y_{0}\right)}{\partial v}(x)=u(x, 0) \text { for } x \in \Gamma_{1}
$$

holds, then (17)-(21) have a unique solution y,z satisfying

$$
y \in B C\left(0,+\infty ; Z_{D}\right) \cap B C^{1}\left(0,+\infty ; H^{-1}(\Omega)\right), z \in H^{1}(0,+\infty ; U) .
$$

Proof. We proceed as in Lasiecka and Triggiani [5] to rewrite the system (17)-21) into an abstract form. Let $A_{D}: D\left(A_{D}\right) \rightarrow X$ be defined by

$$
D\left(A_{D}\right)=H_{0}^{1}(\Omega) \text { and } A_{D} \varphi=-\Delta \varphi, \forall \varphi \in D\left(A_{D}\right)
$$

$A_{D}$ is self-adjoint, positive and boundedly invertible. 
Let $D \in \mathscr{L}\left(L^{2}\left(\Gamma_{1}\right), L^{2}(\Omega)\right)$ be the Dirichlet map given by

$$
g=D v \Longleftrightarrow\left\{\Delta g=0 \text { in } \Omega,\left.g\right|_{\Gamma_{0}}=0,\left.g\right|_{\Gamma_{1}}=v\right\}
$$

Define the operator $B \in \mathscr{L}\left(U, D\left(A_{D}\right)^{\prime}\right)$, by

$$
B u=\mathbf{i} A_{D} D u
$$

Then

$$
B^{*} \varphi=\mathbf{i} D^{*} \varphi=\mathbf{i} \frac{\partial(G \varphi)}{\partial v}
$$

Using these operators, we can formulate (17)-21) as an abstract system of the form

$$
\begin{aligned}
y^{\prime}(t) & =\left(-\mathbf{i} A_{D}-\frac{1}{2} B B^{*}\right) y(t)+B u(t) \\
y(0) & =y_{0} \\
z(t) & =-B^{*} y(t)+u(t)
\end{aligned}
$$

To continue we need the following results.

Lemma 1. (Lasiecka and Triggiani [5]) The operator $A_{1}=-\mathbf{i} A_{D}-\frac{1}{2} B B^{*}$ is the generator of a strongly continuous contraction semigroup $\mathbb{S}$ on $X$.

Lemma 2. (Salamon [13], Weiss \& Tucsnak [20]) Let $u \in H^{2}(0,+\infty ; U)$ and $y_{0} \in X$ satisfy the compatibility condition

$$
A_{1} y_{0}+B u(0) \in X .
$$

Then the initial value problem (29), (30) has a unique solution y given by

$$
y(t)=\mathbb{S}(t) y_{0}+\int_{0}^{t} \mathbb{S}(t-\tau) B u(\tau) d \tau,
$$

which satisfies

$$
y \in C^{1}(0,+\infty ; X) \cap C(0,+\infty ; Z),
$$

where

$$
Z=D\left(A_{1}\right)+(\beta I-A)^{-1} B U .
$$

Lemma 3. Under the assumptions of Lemma 2 we have the identity

$$
\frac{d}{d t}\|y(t)\|^{2}=\|u(t)\|^{2}-\|z(t)\|^{2}
$$

Proof. Taking the inner product of both sides of 29) with $y(t)$, we obtain

$$
\frac{d}{d t}\|y(t)\|^{2}=2 \operatorname{Re}\left\langle A_{1} y(t)+B u(t), y(t)\right\rangle .
$$

From the expression of $A_{1}$, we obtain

$$
\frac{d}{d t}\|y(t)\|^{2}=-\left\|B^{*} u(t)\right\|^{2}+2 \operatorname{Re}\langle B u(t), y(t)\rangle
$$


Using now the formula

$$
\|z(t)\|^{2}=\left\|B^{*} u(t)\right\|^{2}-2 \operatorname{Re}\langle B u(t), y(t)\rangle+\|u(t)\|^{2}
$$

we get the desired identity.

Lemma 4. $B$ is an infinite-time admissible control operator for $\mathbb{S}$.

Proof. Suppose first that

$$
u \in H_{L}^{2}(0,+\infty ; U)=\left\{v \in H^{2}(0,+\infty ; U) ; v(0)=0\right\}
$$

and define $y(t)=\Phi_{t} u$ for all $t \geq 0$. Then $A_{1} y(0)+B u(0) \in X$.

It follows from Lemma 2 that $y(.) \in C^{1}(0,+\infty ; X)$. Integrating the identity in Lemma 3 on $[0, t]$ we get that for all $u \in H_{L}^{2}(0,+\infty ; U)$

$$
\left\|\Phi_{t} u\right\|^{2} \leq \int_{0}^{t}\|u(\tau)\|^{2} d \tau \leq\|u\|_{L^{2}(0,+\infty ; U)}^{2} .
$$

Since $H_{L}^{2}(0,+\infty ; U)$ is dense in $L^{2}(0,+\infty ; U)$, we conclude that $B$ is infnite-time admissible.

Lemma 5. Let $C$ be the restriction of $-B^{*}$ to $D\left(A_{1}\right)$. Then $C$ is an infinite-time admissible operator for $\mathbb{S}$.

Proof. Let $y_{0} \in D\left(A_{1}\right)$ and take $y(t)=\mathbb{S}(t) y_{0}$. It follows from Lemma 2 that $y \in$ $C^{1}(0,+\infty ; X)$. Integrating (36) with $u=0$ on $[0, t]$, we get

$$
\int_{0}^{t}\|z(\tau)\|^{2} d \tau \leq\left\|y_{0}\right\|^{2}
$$

But $z(t)=-B^{*} y(t)=C y(t)$. Therefore the estimate

$$
\int_{0}^{t}\left\|C \mathbb{S}(\tau) y_{0}\right\|^{2} d \tau \leq\left\|y_{0}\right\|^{2}
$$

holds for every $t>0$.

From the previous results, we deduce that the equations (17)-(21) define a well-posed linear system $\Sigma$ with state space $X=H^{-1}(\Omega)$ and input and output space $U=L^{2}\left(\Gamma_{1}\right)$.

The dual system of $\Sigma$ denoted by $\Sigma_{d}$ is described by

$$
\begin{aligned}
y_{d}^{\prime}(t) & =\left(\mathbf{i} A_{D}-\frac{1}{2} B B^{*}\right) y_{d}(t)-B u_{d}(t) \\
z(t) & =B^{*} y_{d}(t)+u_{d}(t)
\end{aligned}
$$

where $u_{d}(t), y_{d}(t)$ and $z_{d}(t)$ are the input, state and output of $\Sigma_{d}$ at some $t \geq 0$.

Proceeding as in the proof of Lemma 3 , one can show that the state trajectory and the output function of $\Sigma_{d}$ satisfy the energy balance equation (36). Hence the conservativity of $\Sigma$. 
Now, notice the following:

- The space $Z=D\left(A_{1}\right)+(\beta I-A)^{-1} D$ introduced in Lemma 1 is given in this case by $Z=D\left(A_{D}\right)+D U$ and coincides with $Z_{D}$.

- The condition (23) is equivalent to $A_{1} y(0)+B u(0) \in X$.

- The transfer function $G(s)$ of $\Sigma$ satisfies, because of the conservativity of $\Sigma$,

$$
\|G(s)\| \leq 1 \text { for all } s \in \mathbb{C}_{0} .
$$

Using these facts together with Lemma 1 Lemma 4 and Lemma 5 we conclude that for every $y_{0} \in Z_{D}$ and every $u \in H^{1}(0,+\infty ; U)$ such that

$$
y_{0}(x)+\frac{\mathbf{i}}{2} \frac{\partial\left(G y_{0}\right)}{\partial v}(x)=u(x, 0) \text { for } x \in \Gamma_{1}
$$

the state trajectory $y$ and the output function $z$ satisfy the smoothness boundedness conditions

$$
y \in B C\left(0,+\infty ; Z_{D}\right) \cap B C^{1}\left(0,+\infty ; H^{-1}(\Omega)\right), z \in H^{1}(0,+\infty ; U) .
$$

(Proposition 4.6 in Weiss and Tucsnak [20]).

Remark 5. Assume the following additional condidtions on the triple $\left\{\Omega, \Gamma_{0}, \Gamma_{1}\right\}$ : there exists a real vector field $h(x) \in\left[C^{1}(\bar{\Omega})\right]^{n}$ such that

$$
\begin{gathered}
\operatorname{Re}\left(\int_{\Omega} H(x) v(x) \cdot \overline{v(x)} d \Omega\right) \geq \rho \int_{\Omega}|v(x)|^{2} d \Omega, \forall v \in\left[L^{2}(\Omega)\right]^{n} \text { for some } \rho>0, \\
\text { where } H(x)=\left(\frac{\partial h_{i}(x)}{\partial x_{j}}\right), i, j=1, \ldots, n
\end{gathered}
$$

and

$$
h . v \leq 0 \text { on } \Gamma_{0} .
$$

Then the semigroup $\mathbb{S}$ is exponentially stable (Lasiecka and Triggiani [5]). Thus, from Russell [11], Tucsnak and Weiss [16], we conclude that the pair $\left(A_{1}, B\right)$ is exactly controllable in finite time and the pair $\left(A_{1}, C\right)$ is exactly observable in finite time.

\section{The Schrödinger Equation with Neumann-Type Boundary Feedback}

In this section, we suppose that the boundary $\Gamma$ is of class $C^{2}$ and satisfies

$$
\Gamma=\Gamma_{0} \cup \Gamma_{1} \text { with } \overline{\Gamma_{1}} \cap \overline{\Gamma_{0}}=\emptyset
$$

where both $\Gamma_{0}$ and $\Gamma_{1}$ are nonempty. 
Let $a($.$) be an L^{\infty}\left(\Gamma_{1}\right)$ such that $a(x) \neq 0$ for all $x \in \Gamma_{1}$.

We are interested in the linear system described by

$$
\begin{gathered}
y_{t}(x, t)=\mathbf{i} \Delta y(x, t) \text { in } \Omega \times(0,+\infty) \\
y(x, 0)=y_{0}(x) \text { in } \Omega \\
y(x, t)=0 \text { on } \Gamma_{0} \times(0,+\infty) \\
\frac{\partial}{\partial v} y(x, t)-\mathbf{i}|a(x)|^{2} y(x, t)=\sqrt{2} a(x) u(x, t) \text { on } \Gamma_{1} \times(0,+\infty) \\
\frac{\partial}{\partial v} y(x, t)+\mathbf{i}|a(x)|^{2} y(x, t)=\sqrt{2} a(x) z(x, t) \text { on } \Gamma_{1} \times(0,+\infty)
\end{gathered}
$$

Remark 6. Lasiecka et al. [9] have considered the system (52)-55] with $u=0$. They have proved under an additional assumption on the triple $\left\{\Omega, \Gamma_{0}, \Gamma_{1}\right\}$ that the energy decays exponentially to zero in the uniform topology of $L^{2}(\Omega)$.

Theorem 5. The equations (52)-(56) determine a conservative linear system $\Sigma$ with input and output space $U=L^{2}\left(\Gamma_{1}\right)$ and state space $X=L^{2}(\Omega)$. If

$$
\left.y_{0} \in Z_{N}=\left\{f \in H_{\Gamma_{0}}^{1}(\Omega): \Delta f \in L^{2}(\Omega), \frac{\partial f}{\partial v} \in a L^{2}\left(\Gamma_{1}\right)\right\}, u \in H^{1}(0,+\infty ; U)\right\}
$$

and the compatibility condition

$$
\frac{\partial}{\partial v} y(x, 0)-\mathbf{i}|a(x)|^{2} y(x, 0)=\sqrt{2} a(x) u(x, 0) \text { for } x \in \Gamma_{1}
$$

holds. Then (52)-(56) have a unique solution $y, z$ satisfying

$$
y \in B C\left(0,+\infty ; Z_{N}\right) \cap B C^{1}\left(0,+\infty ; L^{2}(\Omega)\right), z \in H^{1}(0,+\infty ; U) .
$$

Proof. The equations (52)- 56 can be written as an abstract system of the form (see Lasiecka et al. [9] and Lasiecka and Triggiani [8])

$$
\begin{aligned}
y^{\prime}(t) & =A_{2} y(t)+B u(t) \\
y(0) & =y_{0} \\
z(t) & =-B^{*} y(t)+u(t)
\end{aligned}
$$

where

- $A_{2}=-\mathbf{i} A_{N}-\frac{1}{2} B B^{*}$

- The operator $A_{N}: D\left(A_{N}\right) \rightarrow L^{2}(\Omega)$ is defined by

$$
A_{N} \varphi=-\Delta \varphi, \varphi \in D\left(A_{N}\right)=\left\{\varphi \in Z_{N}: \frac{\partial \varphi}{\partial v}=0 \text { on } \Gamma_{1}\right\}
$$

- $B \in \mathscr{L}\left(U ; D\left(A_{2}\right)^{\prime}\right)$ is defined by

$$
B u=\mathbf{i} \sqrt{2} A_{N} N(a(x) u) ;
$$

- $N \in \mathscr{L}\left(L^{2}\left(\Gamma_{1}\right) ; L^{2}(\Omega)\right)$ is the Neuman map given by

$$
g=N v \Longleftrightarrow\left\{\Delta g=0 \text { in } \Omega,\left.g\right|_{\Gamma_{0}}=0,\left.\frac{\partial g}{\partial v}\right|_{\Gamma_{1}}=v\right\} ;
$$

$-B^{*} \varphi=-\mathbf{i} \sqrt{2} \bar{a}(x) \varphi$. 
Now, the theorem can be established by following the steps of the proof of Theorem 4.

Remark 7. Following Lasiecka et al. [9] and Lasiecka and Triggiani [8], the assertions of Remark 5 hold also for the system (52)-(56).

Acknowledgements. The author is indebted to the anonymous referee for the constructive criticisms and suggestions that led to a significant improvement of the paper.

\section{References}

1. Balakrishnan, A.V.: Applied Functional Analysis, 2nd edn. Springer, Heidelberg (1981)

2. Delfour, M.C.: The linear quadratic optimal control problem with delays in the state and control variables: a state space approach. SIAM J. Control Optim. 24, 835-883 (1986)

3. Flandoli, F., Lasiecka, I., Triggiani, R.: Algebraic Riccati equations with non-smoothing observation arising in hyperbolic and Euler-Bernoulli boundary control problems. Annali Matem. Pura Appl. CLii, 307-382 (1988)

4. Lasiecka, I., Triggiani, R.: A cosine operator approach to modelling $L_{2}\left(0, T ; L_{2}(\Gamma)\right)$ boundary input hyperbolic equations. Appl. Math. Optimiz. 7, 35-83 (1981)

5. Lasiecka, I., Triggiani, R.: Optimal regularity, exact controllability and uniform stabilization of Schrödinger equations with Dirichlet control. Different. and Integral Eqs. 5, 521-535 (1992)

6. Lasiecka, I., Triggiani, R.: $L_{2}(\Sigma)$-regularity of the boundary to boundary operator $B^{*} L$ for hyperbolic and Petrowski PDEs. Abstract and Applied Analysis 19, 1061-1139 (2003)

7. Lasiecka, I., Triggiani, R.: The operator $B^{*} L$ for the wave equation with Dirichlet control. Abstract and Applied Analysis 20, 625-634 (2004)

8. Lasiecka, I., Triggiani, R.: Well-posedness and sharp uniform decay rates at the $L_{2}(\Omega)$-Level of the Schrödinger equation with nonlinear boundary dissipation. J. Evol. Equ. 6, 485-537 (2006)

9. Lasiecka, I., Triggiani, R., Zhang, X.: Global uniqueness, observability and stabilization of non-conservative Schrödinger equation via pointwise Carleman estimates. Part II: $L^{2}(\Omega)$ estimates. J. Inverse Ill-Posed Problems 12, 1-49 (2004)

10. Malinen, J., Staffans, O.J., Weiss, G.: When is a linear system conservative? Quart. Appl. Math. 64, 61-91 (2006)

11. Russell, D.L.: Exact boundary value controllability theorems for wave and heat processes in star complemented regions. In: Roxin, E.O., Sternberg, L. (eds.) Differential Games and Control Theory. Marcel Dekker, New York (1974)

12. Salamon, D.: Control and Observation of Neutral Systems. Pitman, London (1984)

13. Salamon, D.: Infinite dimensional linear systems with unbounded control and observation: a functional analytic approach. Trans. Amer. Math. Soc. 300, 383-431 (1987)

14. Staffans, O.: Well-posed linear systems. Cambridge University Press, Cambridge (2005)

15. Triggiani, R.: A cosine operator approach to modeling $L_{2}\left(0, T ; L_{2}(\Gamma)\right)$-boundary input problems for hyperbolic systems. In: Proceedings of the 8th Conference on Differential Equations and Optimization Techniques. LNCIS, vol. 6, pp. 380-390. University of Würzburg, Germany (1977)

16. Tucsnak, M., Weiss, G.: How to get a conservative well-posed linear system out of thin air. Part II. Controllability and stability. SIAM J. Control Optim. 42, 907-935 (2003) 
17. Washburn, D.: A bound on the boundary input map for parabolic equations with applications to time optimal control. SIAM J. Control Optim. 17, 652-671 (1979)

18. Weiss, G.: Regular linear systems with feedback. Mathematics Control, Signal \& Systems 7 , 25-57 (1994)

19. Weiss, G., Staffans, O., Tucsnak, M.: Well-posed linear systems - a survey with emphasis on conservative systems. Internat. J. Appl. Math. Comput. Sci. 11, 7-34 (2001)

20. Weiss, G., Tucsnak, M.: How to get a conservative well-posed linear system out of thin air. Part I. Well-posedness and energy balance. ESAIM. Control, Optim. Calc. Var. 9, 247-274 (2003) 